\title{
Editorial
}

Skin

Appendage

Disorders
Skin Appendage Disord 2015;1:I

DOI: $10.1159 / 000380774$
Published online: February 19, 2015

\section{Another Medical Journal or Filling a Gap?}

Looking at the first issue of an ambitious new publication in the field of dermatology, the main question that needs a clear answer is whether we really need Skin Appendage Disorders or whether it is just one of the many new medical journals that seem to lack clear direction and fail to provide worthwhile information. To give a clear answer, we have to point out that Skin Appendage Disorders is a journal dedicated exclusively to hair, nails and skin glands. It aspires to deal with every aspect of all disorders that affect the skin appendages.

But do we really need a journal focusing on hair, nails and skin glands? Numerous reliable and reputable medical journals in the field of dermatology include articles on these topics. To address this question, we should remind ourselves of just two things: first, the huge volume of medical literature being published nowadays makes it really difficult for medical professionals to collect information on a specific field, and it has become nearly impossible to assess the validity of this information. Second, skin appendage disorders require specific training in diagnosis and treatment as innovative pharmaceutical therapies and novel surgical techniques continue to increase steadily.

Skin Appendage Disorders will present both research and review articles that reflect the 'state-of-the-art' knowledge in these specific fields. The journal will also provide its reader with all the recent information about novel diagnostic procedures and innovative therapeutic approaches. Furthermore, articles discussing important basic research will explore and provide valuable insights in future developments in the field.

Consequently, Skin Appendage Disorders aims to be a journal of reference for any scientist, dermatologist or other medical professional who wishes to focus on hair, nails and skin glands. Reputable scientists and opinion makers in this field have been chosen to form the editorial board and will ensure that the information provided in this journal will always be valid and reliable.

To conclude, it will ultimately be up to its readers to decide whether Skin Appendage Disorders deserves to succeed. This is likely to happen if they assess it positively in order to integrate it in the journals which regularly refer to it. This is our goal and we intend to work hard to achieve it!

Dimitris Rigopoulos Antonella Tosti 